University of Wollongong

Research Online

Faculty of Business - Papers (Archive)

Faculty of Business and Law

$1-1-2016$

Economic growth and abatement activities in a stochastic environment: a multi-objective approach

Simone Marsiglio

University of Wollongong, simonem@uow.edu.au

Davide La Torre

University of Milan

Follow this and additional works at: https://ro.uow.edu.au/buspapers

Part of the Business Commons

Research Online is the open access institutional repository for the University of Wollongong. For further information contact the UOW Library: research-pubs@uow.edu.au 


\title{
Economic growth and abatement activities in a stochastic environment: a multi- objective approach
}

\author{
Abstract \\ We analyze the effect of uncertainty on economic growth and environmental quality in a concave \\ stochastic bicriteria dynamic problem, solved by means of scalarization techniques and the Hamilton- \\ Jacobi-Bellman equation. Specifically, we focus on an endogenous growth model with purposive \\ abatement activities, where environmental quality, subject to random shocks, is merely a source of utility \\ and does not play any productive role. We show that even in the absence of direct (environmental) \\ productivity effects, along the optimal path production results enhanced by an indirect productivity effect, \\ which transforms environmental quality into the engine of growth. However, this does not prevent \\ uncertainty to dampen economic and environmental performance, leading to a lower social welfare than \\ in a deterministic framework.

\section{Disciplines} \\ Business

\section{Publication Details} \\ Marsiglio, S. \& La Torre, D. (2018). Economic growth and abatement activities in a stochastic \\ environment: a multi-objective approach. Annals of Operations Research, 267 (1-2), 321-334.
}




\title{
Economic Growth and Abatement Activities in a Stochastic Environment: a Multi-Objective Approach*
}

\author{
Simone Marsiglio ${ }^{\dagger} \quad$ Davide La Torre ${ }^{\ddagger}$ \\ Forthcoming in Annals of Operations Research
}

\begin{abstract}
We analyze the effect of uncertainty on economic growth and environmental quality in a concave stochastic bicriteria dynamic problem, solved by means of scalarization techniques and the HamiltonJacobi-Bellman equation. Specifically, we focus on an endogenous growth model with purposive abatement activities, where environmental quality, subject to random shocks, is merely a source of utility and does not play any productive role. We show that even in the absence of direct (environmental) productivity effects, along the optimal path production results enhanced by an indirect productivity effect, which transforms environmental quality into the engine of growth. However, this does not prevent uncertainty to dampen economic and environmental performance, leading to a lower social welfare than in a deterministic framework.
\end{abstract}

Keywords: Economic Growth, Environmental Quality, Uncertainty, Multi-objective Optimization JEL Classification: O40, O41, Q50, Q56

\section{Introduction}

After decades of debates among policymakers and researchers, a considerable interest on environmental problems has finally grown, as recently confirmed by the final report of the United Nations Conference on Sustainable Development (UNEP, 2012). In particular, an urgent matter for any authority and institution responsible for environmental policy is to understand and predict how environmental quality will tend to evolve in the future, because of production, consumption and preservation activities. Uncertainty in this context plays a fundamental role: very little is known on how climate change will affect economic processes and our wellbeing, thus it is essential to understand how we should respond to unclear future events. Should we increase green investments to prevent eventual larger environmental losses? Should we reduce economic activity as a precaution? How will uncertainty affect wellbeing? Despite the importance of such questions, very few studies analyze the effect of environmental uncertainty on economic outcomes 1 . Existing works focus on the production side, looking at how uncertainty on the production process affects economic agents' decisions (Soretz, 2003; 2004; 2007), or show how uncertainty on the evolution of pollution may generate different development paths (Kijima et al., 2011; Privileggi and Marsiglio, 2013). To the best of

\footnotetext{
${ }^{*}$ We are grateful to two anonymous referees for their constructive comments on an earlier draft which helped to substantially improve our paper.All remaining errors and omissions are our own sole responsibility.

${ }^{\dagger}$ University of Wollongong, School of Accounting, Economics and Finance, Northfields Avenue, Wollongong 2522 NSW, Australia. Contact: simonem@uow.edu.au.

${ }^{\ddagger}$ University of Milan, Department of Economics, Management and Quantitative Methods, Milan, Italy, and Khalifa University, Department of Applied Mathematics and Science, Abu Dhabi, UAE Contact: davide.latorre@unimi.it

${ }^{1}$ Even if the works focusing also on the environment are limited, there is an abundant number of papers analyzing the relation between growth and uncertainty (see among others, Aghion et al., 2010; De Hek, 1999; Steger, 2005; Walde, 2005). Since our main goal is to understand the environmental implications on uncertainty, in the paper we do not specifically relate to this branch of literature.
} 
our knowledge, apart from Athanassoglou and Xepapadeas (2012) and La Torre et al. (2016) who analyze a pollution control problem under uncertainty without modeling the related macroeconomic effects, how uncertainty affects the trade off between environmental and economic performance in an economic growth setup has never been analyzed thus far.

In this paper we try to analyze how environmental shocks affect economic outcomes and environmental policy. Thus, our work is strictly related to that large share of the literature analyzing the economicenvironmental trade off in deterministic frameworks (see Xepapadeas, 2003, for a survey). In order to keep the analysis as simple as possible we consider a one sector model of endogenous growth. In such a context, the (deterministic) literature shows that economic growth and environmental preservation are to a large extent compatible, and sustainable balanced growth path (BGP) equilibria may exist. How environmental policy relates to economic growth is more complex (see Smulders, 1999). Indeed, environmental preservation requires to divert some resources from growth enhancing activities (crowding out effect), and at the same time it may lead to productivity improvements (productivity effect). Thus, growth and environment will go hand-in-hand if the productivity effect is large enough to more than compensate the crowding out effect. Whether a similar result may hold or not even in a stochastic context requires further investigation.

This is exactly the focus of this paper, in which we analyze an almost standard economic growth model with environmental interaction in presence of uncertainty in environmental dynamics. As standard in this type of literature the objective function, representing social welfare, depends upon two different and conflicting variables, consumption and environmental quality. By relying on an additive specification of the utility function, the model gives rise to a multicriteria optimization problem, in which the two criteria are scalarized through a parameter representing the green preference parameter. This formulation is particularly interesting since it allows us to show that traditional economic growth and environment models (under some specific conditions) can be interpreted as multicriteria problems, partially bridging the gap between economics and the operational research literatures. Indeed, to the best of our knowledge, despite the similarities in the approach, very limited are the applications of multicriteria techniques in economics (a noticeable exception is represented by Colapinto et al., 2015).

Since in the economics literature, multicriteria techniques are not widely known, it may be useful to recall some basic result which will be useful in our analysis (see Sawaragi et al., 1985). In an abstract setting, a finite-dimensional multicriteria optimization problem can be stated as follows:

$$
\max J(x), \quad x \in X
$$

where $(X,\|\cdot\|)$ is a Banach space and $J: X \rightarrow \mathbb{R}^{p}$ is a vector-valued functional, and $\mathbb{R}^{p}$ is ordered by the Pareto cone $\mathbb{R}_{+}^{p}$. A point $x \in X$ is said to be Pareto optimal or efficient if $J(x)$ is one of the maximal elements of the set of achievable values $J(X)$. Thus a point $x$ is Pareto optimal if it is feasible and, for any possible $x^{\prime} \in X, J(x) \leq_{\mathbb{R}_{+}^{p}} J\left(x^{\prime}\right)$ implies $x=x^{\prime}$. In a more synthetic way, a point $x \in X$ is said to be Pareto optimal if $\left(J(x)+\mathbb{R}_{+}^{p}\right) \cap J(X)=\{J(x)\}$.

Among the different methods proposed in the operational research literature, scalarization is probably the simplest and one of the most widely used techniques in order to identify Pareto optimal solutions. By relying on scalarization techniques it is possible to characterize Pareto optimal solutions for any multicriteria problem (1) by solving the following scalar problem:

$$
\max \beta J(x), \quad x \in X
$$

where $\beta$ is a vector taking values in the interior of $\mathbb{R}_{+}^{p}$, namely $\beta \in \operatorname{int}\left(\mathbb{R}_{+}^{p}\right)$. Since the Pareto optimal solution depends on $\beta$, by varying $\beta$ it is possible to obtain different Pareto optimal points. Now let us suppose that each component $J_{i}$ is concave, thus $J$ is a vector-valued concave functional. Under this assumption, the scalarized problem (2) is also concave. This means that we can find Pareto optimal points of a concave problem by solving a concave scalar optimization problem, and for each $\beta \in \operatorname{int}\left(\mathbb{R}_{+}^{p}\right)$ different 
Pareto optimal points can be obtained. For concave problems the converse of this result is only partially true, since for every Pareto optimal point $\bar{x}$, there is a nonzero $\bar{\beta} \in \mathbb{R}_{+}^{p}$ such that $\bar{x}$ is a solution of the scalarized problem $(2)$ with $\beta=\bar{\beta}$.

This brief paper proceeds as follows. Section 2 presents our bicriteria model, which consists of an endogenous growth model with purposive abatement activities where environmental quality, subject to random shocks, is a mere source of utility with no productive role. The social planner tries to achieve two conflicting goals, an economic and an environmental goal, by taking into account that producing more output in order to allow higher consumption levels tends to increase polluting emissions which lower environmental quality. Environmental quality is modeled as a stock variable negatively affected by flow emissions which are a by-product of economic activities (Brock and Taylor, 2005). Slightly differently from most of the papers in the literature which focus on the optimal amount of resources to allocate to environmental protection (Chevé, 2000; Shieh et al., 2001), we will focus on the determination of optimal amount of emissions (Bartz and Kelly, 2008; Stokey, 1998), which from a regulator point of view may be more relevant and interesting. Section 3 derives the optimal solution by identifying an explicit solution for the Hamilton-Jacobi-Bellman equation, which allows to characterize the optimal dynamic paths of both control and state variables. We show that economic performance may be enhanced by the diversion of resources from the capital to environmental sector, which eventually transforms environmental quality in the engine of long run growth; this however does not prevent uncertainty to restrain both economic and environmental performance. Section 4 focuses on a specific case of the model which allows to explicitly analyze how the two conflicting criteria depend on the green preference parameter, which represents the scalarization parameter. This permits to explicitly characterize the Pareto frontier in order to look separately at each of two criteria, but also to derive social welfare in order to look at the scalarized criterion. Section 5 contains concluding remarks and proposes directions for future research.

\section{The Model}

We consider a Ramsey-type (1928) model of optimal growth where the social planner, by taking into account economic and environmental constraints, chooses the level of consumption, $c(t)$, and the share of emission to abate, $u(t)$, in an attempt to simultaneously achieve two conflicting goals, related to economic and environmental performance respectively. The planner's objective function is thus characterized by a twocriteria functional, in which each criterion is represented by the infinite discounted ( $\rho$ is the pure rate of time preference) sum of the instantaneous utilities associated with the respective goal, given by consumption and environmental quality, $E(t)$. The instantaneous utility functions associated with consumption and environmental quality are assumed to be isoelastic and given by $U(c(t))=\frac{c(t)^{1-\theta}-1}{1-\theta}$ and $u(E(t))=\frac{E(t)^{1-\nu}-1}{1-\nu}$, respectively, where $\theta>0$ and $\nu>0$ denote the inverse of the intertemporal elasticity of substitution in consumption and environment. Capital, $k(t)$, accumulation is given by the difference between net (of depreciation, $\delta$ ) production of the unique final good, consumption and (emission) abatement activities, $a(t)$ : $\dot{k}(t)=y(t)-\delta k(t)-c(t)-a(t)$. Production is assumed to be linear in capital, $y(t)=A k(t)$, where $A$ is a scale parameter. Abatement activities use a certain share of output, $a(t)=x(t) y(t)$, where $x(t)$ is the cost function which, as in Bartz and Kelly (2008), is assumed to be nonlinear in the share of abated emissions, $u(t) \in[0,1]$, and to take the following suitable form 2 , $x(t)=1-\left(1-u_{t}\right)^{\epsilon}$ with $\epsilon>0$. Economic activities generate pollution, $p(t)$, and emissions are assumed to depend on capital: $p(t)=\eta k(t)^{\phi}$, where $\phi, \eta>0$ are technological parameters measuring the degree of pollution intensity. Purposive abatement activities reduce a certain share of emissions, $u(t) p(t)$, such that the resulting unabated pollution is $b(t)=[1-u(t)] p(t)$. Thus, environmental quality evolves according to the difference between the pollution absorption capacity,

\footnotetext{
${ }^{2}$ Bartz and Kelly (2008) assume that the function is convex, i.e. $\epsilon>1$, while we do not impose a priori any restriction on the shape of the cost function, and we allow for the possibility that this is even concave, $\epsilon<1$. However, the parametric restriction imposed by our solution will preclude us to consider the convex case.
} 
assumed to be proportional to environmental quality ${ }^{3}$ ( $\mu$ is the natural rate of pollution absorption), and unabated emissions: $\dot{E}(t)=\mu E(t)-b(t)$. However, the environment is also subject to random shocks driven by a Brownian motion (as in Kijima et al., 2011).

The social planner's problem consists thus of choosing $c(t)$ and $u(t)$ in order to maximize the following concave bicriteria functional, given the capital and environmental dynamic constraints, and initial conditions, $k_{0}>0$ and $E_{0}>0$ :

$$
\begin{aligned}
\max _{c(t), u(t)} & \left(\mathbb{E}\left[\int_{0}^{\infty}\left(\frac{c(t)^{1-\theta}-1}{1-\theta}\right) e^{-\rho t} d t\right], \mathbb{E}\left[\int_{0}^{\infty}\left(\frac{E(t)^{1-\nu}-1}{1-\nu}\right) e^{-\rho t} d t\right]\right) \\
\text { s.t. } & \dot{k}(t)=[1-u(t)]^{\epsilon} A k(t)-\delta k(t)-c(t) \\
& d E(t)=\left\{\mu E(t)-[1-u(t)] \eta k(t)^{\phi}\right\} d t+\sigma E(t) d B(t)
\end{aligned}
$$

where $\sigma \geq 0$ is the variance parameter and $d B(t)$ is the increment of a Wiener process. Uncertainty about the health of the environment, by determining environmental quality, directly affects the objective function, but does not play any role in the economic production process. Thus, abatement activities aim only to reduce the impact of eventual negative environmental shocks on agents's welfare (generating a crowding out effect and no productivity effect). Note that if no resources are allocated to abatement activities (i.e. $u=0$ ), environmental quality will get worse and worse as a consequence of economic growth5; if all resources are devoted to environmental preservation (i.e. $u=1$ ), nothing will be left for consumption. Thus, the optimal choice will require $0<u<1$.

The previous bicriteria problem can be simplified by means of a linear scalarization technique as follows:

$$
\begin{aligned}
\max _{c(t), u(t)} & \beta_{1} \mathbb{E}\left[\int_{0}^{\infty}\left(\frac{c(t)^{1-\theta}-1}{1-\theta}\right) e^{-\rho t} d t\right]+\beta_{2} \mathbb{E}\left[\int_{0}^{\infty}\left(\frac{E(t)^{1-\nu}-1}{1-\nu}\right) e^{-\rho t} d t\right] \\
\text { s.t. } & \dot{k}(t)=[1-u(t)]^{\epsilon} A k(t)-\delta k(t)-c(t) \\
& d E(t)=\left\{\mu E(t)-[1-u(t)] \eta k(t)^{\phi}\right\} d t+\sigma E(t) d B(t),
\end{aligned}
$$

where $\beta_{1}$ and $\beta_{2}$ are two strictly positive parameters, measuring the weight of each goal in the planner's problem. As mentioned in the previous section, by varying the vector $\left(\beta_{1}, \beta_{2}\right)$ in $\operatorname{int}\left(\mathbb{R}_{+}^{2}\right)$, we can obtain different Pareto optimal solutions for the above bicriteria problem; we will be back to this point in section 4 where we will determine the efficient frontier and compare the optimal value of the two criteria with the value of the scalarized criterion. By defining $\beta=\frac{\beta_{2}}{\beta_{1}}$, and using the linearity properties of the expected value and of the integral operators, the scalarized problem turns out to be completely equivalent to the following:

$$
\begin{aligned}
\max _{c(t), u(t)} & W=\mathbb{E}\left[\int_{0}^{\infty}\left(\frac{c(t)^{1-\theta}-1}{1-\theta}+\beta \frac{E(t)^{1-\nu}-1}{1-\nu}\right) e^{-\rho t} d t\right] \\
\text { s.t. } & \dot{k}(t)=[1-u(t)]^{\epsilon} A k(t)-\delta k(t)-c(t) \\
& d E(t)=\left\{\mu E(t)-[1-u(t)] \eta k(t)^{\phi}\right\} d t+\sigma E(t) d B(t),
\end{aligned}
$$

\footnotetext{
${ }^{3}$ By following the traditional approach in the growth and environment literature, we assume that environmental quality is unbounded meaning that "ecological processes are modelled as growth and depletion of a renewable resources" (Smulders, 1999). This specification implies that we completely ignore the role of non-renewable resources in order to emphasize pollution problems. Different formulations of the pollution absorption (or renewal) capacity of the environment have been proposed in order to analyze issues related to material and energy scarcity; see for example earlier works in the field (Dasgupta and Heal, 1978). Note that our formulation of environmental quality and pollution is consistent with Brock and Taylor's (2005), where pollution is a by-product of productive activities meaning that when output is zero (infinite) pollution is zero (infinite) too.

${ }^{4}$ Differently from Kijima et al. (2011), where the environment is subject to completely exogenous shocks, in our framework the planner by choosing the share of emissions to abate endogenously determines the trend of the stochastic process affecting environmental quality.

${ }^{5}$ Notice that in the $u=0$ case, for sufficiently small levels of capital environmental quality might initially improve due to pollution absorption; however, as capital keeps growing it will decrease.
} 
Note that the formulation of the problem (9), (10) and (11) represents the traditional specification used in the economics literature. Indeed, by scalarizing the two criteria in a single criterion, the objective function represents the social welfare, $W$ (defined as the expected infinite discounted sum of instantaneous utilities), in a situation in which the utility function depending on both consumption and environmental quality, is separable in its two arguments, $U(c(t), E(t))=\frac{c(t)^{1-\theta}-1}{1-\theta}+\beta \frac{E(t)^{1-\nu}-1}{1-\nu}$, and $\beta$ represents the green preference parameter, measuring the relative weight of the environment in the planner's preferences. A similar approach, based on a scalarization technique, is used also in Colapinto et al. (2015) in order to analyze the intergenerational equity and sustainability issues in a macroeconomic setting without capital and environment interaction. To the best of our knowledge, our work is the first paper analyzing analytically a stochastic multicriteria problem in economics.

\section{Equilibrium}

By defining $J(k, E)$ as the maximum expected value associated with the above stochastic optimization problem, the Hamilton-Jacobi-Bellman (HJB) equation is:

$$
\rho J=\max _{c, u}\left\{\frac{c^{1-\theta}-1}{1-\theta}+\beta \frac{E^{1-\nu}-1}{1-\nu}+J_{k}\left[(1-u)^{\epsilon} A k-\delta k-c\right]+J_{E}\left[\mu E-(1-u) \eta k^{\phi}\right]+\frac{1}{2} \sigma^{2} J_{E E} E^{2}\right\}
$$

First order necessary and sufficient conditions with respect to $c$ and $u$ read as:

$$
\begin{aligned}
c & =J_{k}^{-\frac{1}{\theta}} \\
1-u & =\epsilon^{-\frac{1}{\epsilon-1}} \eta^{\frac{1}{\epsilon-1}} A^{-\frac{1}{\epsilon-1}} J_{E}^{\frac{1}{\epsilon-1}} J_{k}^{-\frac{1}{\epsilon-1}} k^{\frac{\phi-1}{\epsilon-1}},
\end{aligned}
$$

which substituted back into the HJB equation yield:

$$
\begin{aligned}
\rho J+\frac{1-\nu+\beta(1-\theta)}{(1-\theta)(1-\nu)}= & \frac{\theta}{1-\theta} J_{k}^{-\frac{1-\theta}{\theta}}+\beta \frac{E^{1-\nu}}{1-\nu}-\epsilon^{-\frac{\epsilon}{\epsilon-1}}(\epsilon-1) \eta^{\frac{\epsilon}{\epsilon-1}} A^{-\frac{1}{\epsilon-1}} J_{E}^{\frac{\epsilon}{\epsilon-1}} J_{k}^{-\frac{1}{\epsilon-1}} k^{\frac{\phi \epsilon-1}{\epsilon-1}}+ \\
& -\delta J_{k} k+\mu J_{E} E+\frac{1}{2} \sigma^{2} J_{E E} E^{2}
\end{aligned}
$$

By applying the guess and verify method, it is possible to show that a closed form solution for such a problem exists under a particular combination of parameter values (the proofs of the following propositions are presented in appendix A). Note that whenever these conditions are not met we could alternatively determine an approximate solution by means of a goal programming model as in Aouni et al. (2015).

Proposition 1. Assume that $\phi=\frac{1-\theta}{\epsilon}$ and $\epsilon=1-\nu$; then the value function is given by:

$$
J(k, E)=\xi_{1} k(t)^{1-\theta}+\xi_{2} E(t)^{\epsilon}+\xi_{3},
$$

where:

$$
\begin{array}{r}
\xi_{1}=\frac{\theta^{\theta}}{(1-\theta)(\rho+\delta)^{\theta}}, \quad \xi_{3}=-\frac{\epsilon+\beta(1-\theta)}{\rho \epsilon(1-\theta)}, \\
\frac{\beta}{\epsilon}+\xi_{2}\left[\mu \epsilon-\frac{1}{2} \sigma^{2} \epsilon(1-\epsilon)-\rho+(1-\epsilon) \eta^{-\frac{\epsilon}{1-\epsilon}} A^{\frac{1}{1-\epsilon}} \xi_{2}^{-\frac{1}{1-\epsilon}}(1-\theta)^{\frac{1}{1-\epsilon}} \xi_{1}^{\frac{1}{1-\epsilon}}\right]=0 .
\end{array}
$$

Since the instantaneous utility function is separable in its arguments, the value function is separable in the state variables too. Note that the imposed parametric restriction is compatible only with the concave abatement cost case $(\epsilon<1)$; indeed, in the convex case $(\epsilon>1), \nu$ would turn out to be negative. It 
also requires that $\theta<1$ in order for $\phi$ to be positive $e^{6}$. This latter condition $(\theta<1)$ is standard both in the stochastic growth literature and in the growth and environment literature. In a stochastic growth framework the inverse of the intertemporal elasticity of substitution is traditionally required to be smaller than the capital share, which in our case is equal to the unity (Smith, 2007). In a growth and environment context the inverse of the intertemporal elasticity of substitution is thought to be smaller than one in order to allow for win-win outcomes (Mohtadi, 1996). The other condition $(\epsilon<1)$ is not so standard but is needed in order to impose sufficient convexity to our problem; its requirement that the abatement cost function is concave implies that reducing pollution becomes relatively cheaper as the share of abated pollution gets larger, which is consistent with the idea of increasing returns to scale in the abatement proces: $7^{7}$ (Andreoni and Levinson, 2001). Note that in Proposition 1 we are able to explicitly determine only two out of the three coefficients in the value function $(16)$. In particular, the required parametric restrictions do not even allow us to conclude whether a solution for the coefficient $\xi_{2}$ exists or not. It is however, possible to show that, provided that an additional condition is met, a unique and positive solution exists.

Proposition 2. Assume that $\rho>\mu \epsilon$. Then there exists a unique positive $\xi_{2}$ solution to the equation $g\left(\xi_{2}\right)=\frac{\beta}{\epsilon}+\xi_{2}\left[\mu \epsilon-\frac{1}{2} \sigma^{2} \epsilon(1-\epsilon)-\rho+(1-\epsilon) \eta^{-\frac{\epsilon}{1-\epsilon}} A^{\frac{1}{1-\epsilon}} \xi_{2}^{-\frac{1}{1-\epsilon}}(1-\theta)^{\frac{1}{1-\epsilon}} \xi_{1}^{\frac{1}{1-\epsilon}}\right]=0$. Furthermore $\frac{\partial \xi_{2}}{\partial \sigma}<0$ and $\frac{\partial \xi_{2}}{\partial \beta}>0$.

In order for a unique coefficient $\xi_{2}$ to exist and to be positive, we need the rate of time preference to be large enough $(\rho>\mu \epsilon)$. Such a condition is likely to be met in reality since the term $\mu \epsilon$ may turn out to be very small; indeed, the natural rate of pollution absorption is estimated to be quite small, and multiplied by $\epsilon$ which is smaller than unity, it gets even smaller. This additional parametric restriction allows us to be sure that even if we do not have an explicit expression for $\xi_{2}$ the value function (16) is well defined. From now onward we restrict our analysis to the situation in which the conditions in both Proposition 1 and Proposition 2 are simultaneously met; note that in such a case, sufficiency requires that $\phi \in\left(1, \frac{1}{\epsilon}\right)$. Given such a formulation of the value function, it is straightforward to derive the explicit dynamic paths of the control and state variables, by substituting the relevant expression from (16) back into (13), (14), (10) and (11).

Proposition 3. The optimal rules for consumption and share of abated emissions are given by:

$$
\begin{aligned}
c(t) & =\frac{\rho+\delta}{\theta} k(t), \\
1-u(t) & =\varphi E(t) k(t)^{-\frac{1-\theta}{\epsilon}},
\end{aligned}
$$

while the optimal dynamic paths of capital and environmental quality are:

$$
\begin{aligned}
\dot{k}(t) & =A \varphi^{\epsilon} E(t)^{\epsilon} k(t)^{\theta}-\Psi k(t), \\
d E(t) & =(\mu-\eta \varphi) E(t) d t+\sigma E(t) d B(t)
\end{aligned}
$$

where $\Psi=\frac{\rho+\delta(1+\theta)}{\theta}$ and $\varphi=\eta^{-\frac{1}{1-\epsilon}}(\rho+\delta)^{-\frac{\theta}{1-\epsilon}} \theta^{\frac{\theta}{1-\epsilon}} A^{\frac{1}{1-\epsilon}} \xi_{2}^{-\frac{1}{1-\epsilon}}$.

Equation (17) highlights the existence of a linear relationship between consumption and capital; this result is consistent with what is traditionally shown in the stochastic growth literature 8 (see Smith, 2007).

\footnotetext{
${ }^{6}$ Note that if the intertemporal elasticity of substitution in consumption, $\theta$, and environment, $\nu$, coincide $(\theta=\nu)$, then the parameter restrictions imposed by Proposition 1 may appear less stringent; indeed, we will simply need that $\phi=1$ and $\epsilon=1-\theta$. Since this represents a specific case of our model it seems more convenient to present it in its most general form.

${ }^{7}$ Moreover, note that if $\epsilon>1$ then the production function at equilibrium will be characterized by increasing returns to scale with respect to environmental quality; see equation 19 .

${ }^{8}$ Similar results are obtained in growth models with productivity (Smith, 2007; Bucci et al., 2011) and demographic (Marsiglio and La Torre, 2012; Marsiglio, 2014) shocks.
} 
Equation (18) shows that the share of abated emissions in absence of shocks is constant since, from equation (11), along a BGP equilibrium we would need $\gamma_{E}=\phi \gamma_{k}$, where $\gamma_{z}$ denotes the growth rate of the variable $z(t)$ (see Proposition 4); however, random shocks may lead this share to change over time. The optimal dynamics of capital and environmental quality, in (19) and (20) deserve some explanation. Even if the dynamic equations of capital and environmental quality (equations (10) and (11) show that environmental quality does not have any impact on capital accumulation (no productivity effect) while capital directly affects the environment through the pollution channel, the optimal dynamics of $k$ and $E$ state exactly the opposite. Indeed, once the control variables are optimally chosen, the environment is no longer affected by the evolution of capital while capital is crucially affected by the (stochastic) environmental quality, implying that at equilibrium environmental quality generate a sort of indirect productivity effect allowing to compensate the setting of diminishing returns into capital accumulation (see equation (19)). Moreover, if environmental protection activities are sufficiently effective $(\mu>\eta \varphi)$, environmental quality will tend to improve over time, becoming the engine of long run growth?

Indeed, in a deterministic framework where uncertainty does not play any role $(\sigma=0)$, it is straightforward to solve for the path of capital and derive the BGP equilibrium 10 .

Proposition 4. In absence of uncertainty (i.e., $\sigma=0$ ), if $\mu>\eta \varphi$, along the BGP equilibrium environmental and economic growth rates will be strictly positive and respectively given by:

$$
\begin{aligned}
\gamma_{E} & =\mu-\eta \varphi \\
\gamma & =\gamma_{k}=\gamma_{y}=\gamma_{c}=\frac{\epsilon}{1-\theta} \gamma_{E}=\frac{\epsilon(\mu-\eta \varphi)}{1-\theta} .
\end{aligned}
$$

Proposition 4 states that along the BGP abatement activities, if sufficiently effective, allow environmental quality to indefinitely increase over time, becoming the engine of long run growth. Note that since environmental policy affects $\varphi$ (see equation (18), and compare (11) and (20)), it might be used to achieve both to a healthier environment $\left(\frac{\partial \gamma_{E}}{\partial \varphi}=-\eta<0\right)$ and faster growth $\left(\frac{\partial \gamma}{\partial \varphi}=-\frac{\epsilon \eta}{1-\theta}<0\right)$, resulting in a win-win outcome leading to steady welfare improvements. This means that even without (direct) productive effects in a canonical one sector endogenous growth model it may be possible for growth and environment to go hand-in-hand 11 . Specifically, the mechanism that allows to overcome the crowding out effect is related to the existence at equilibrium of an indirect productivity effect leading environmental quality to sustain long run growth. However, despite such a growth potential as soon as uncertainty is introduced $(\sigma>0)$ both economic and environmental performances will be reduced. This can be directly seen by contrasting the stochastic and the deterministic version of the model. In the following, for any variable $x(t)$ we denote its deterministic outcome by $\bar{x}(t)$. From Jensen's inequality, the random process $E(t)$ under the concave transformation $E(t)^{\epsilon}$ (note that $\epsilon<1$ ) will always show $\mathbb{E}\left[E(t)^{\epsilon}\right] \leq \bar{E}(t)^{\epsilon}$. It then directly follows that:

$$
\begin{aligned}
\mathbb{E}\left[k(t)^{1-\theta}\right] & =e^{-(1-\theta) \Psi t}\left[(1-\theta) A \varphi^{\epsilon} \int_{0}^{t} e^{(1-\theta) \Psi v} \mathbb{E}\left[E(t)^{\epsilon}\right] d v+k_{0}^{1-\theta}\right] \\
& \leq e^{-(1-\theta) \Psi t}\left[(1-\theta) A \varphi^{\epsilon} \int_{0}^{t} e^{(1-\theta) \Psi v} \bar{E}(t)^{\epsilon} d v+k_{0}^{1-\theta}\right]=\bar{k}(t)^{1-\theta} .
\end{aligned}
$$

Given the parameter restrictions imposed on $(16)$, these relationships imply that also social welfare, from equation (9), will tend to drop in the stochastic case.

\footnotetext{
${ }^{9} \mathrm{By}$ comparing the dynamics of capital and environment without policy intervention (equations 10 and 11 ) with their corresponding dynamics after the environmental policy has been optimally implemented (equations (19) and (20)), it is clear that policy intervention allows environmental quality to substitute capital as the engine of sustained growth.

${ }^{10}$ Note that being the model of the AK-type no transitional dynamics exists, thus we cannot characterize out of BGP dynamics and implications of the model.

${ }^{11}$ Similar conclusions can be derived in more complex frameworks but not in basic setups comparable to ours (see Ricci, 2007, for a detailed survey).
} 
Proposition 5. Uncertainty tends to dampen both environmental quality and capital (namely $\mathbb{E}\left[E(t)^{\epsilon}\right] \leq$ $\bar{E}(t)^{\epsilon}$ and $\mathbb{E}\left[k(t)^{1-\theta}\right] \leq \bar{k}(t)^{1-\theta}$, respectively), reducing social welfare (i.e., $\mathbb{E}[W] \leq \bar{W}$ ).

In our growth and environment setting, Proposition 5 shows that the levels of capital and environmental quality in a stochastic framework will generally be smaller than their counterparts in the deterministic case $\epsilon^{12}$. The result that capital accumulation is reduced as a response to increasing uncertainty is consistent with previous works on stochastic endogenous growth (Bucci et al., 2011; Marsiglio and La Torre, 2012); comparing the result that also environmental quality is reduced by uncertainty with previous literature is more difficult since these works assume that pollution is stochastic as a consequence of randomness in production 13 (Soretz, 2003; 2007). Moreover, differently from these studies, environmental quality which represents the source of uncertainty directly affects welfare, thus the outcome in Proposition 5 is not so obvious a priori. However, even if some resources are allocated to preserve the environment, uncertainty about environmental processes negatively affects both the economy and the environment. Diverting resources from the physical to the environmental sector transforms the production process from a polluting (capital) to a green (environment) growth source, but this does not prevent social welfare to fall due to uncertainty.

Note that our results are derived for a given value of the green preference parameter $\beta$, which in our growth and environment context can be taken as exogenously given. However, in the bicriteria formulation of the problem $\beta$ which represents the scalarizing parameter plays a very important role. Indeed, by varying $\beta$ between zero and infinity it is possible to change the relative weight of the two criteria, and this might allow us to derive different optimal solutions. Each of these solution will be optimal in Pareto sense and will belong to the efficient frontier of the scalarized bicriteria problem (9), (10) and (11).

\section{The Efficient Frontier and Social Welfare}

In order to determine a qualitative representation of the Pareto frontier, we need the exact expressions of the optimal values of both criteria $J_{1}$ and $J_{2}$ and how they depend on the scalarization parameter $\beta$. With some simple algebra, we can obtain the following expressions for the optimal values of the two criteria:

$$
\begin{aligned}
& J_{1}=\frac{\left(\frac{\rho+\delta}{\theta}\right)^{1-\theta} E_{0}^{\epsilon} A \varphi^{\epsilon}}{[\rho-\epsilon(\mu-\eta \varphi)][(1-\theta) \Psi+\rho]}+\frac{\left(\frac{\rho+\delta}{\theta}\right)^{1-\theta} E_{0}^{\epsilon} k_{0}^{1-\theta}}{(1-\theta)[(1-\theta) \Psi+\rho]}-\frac{1}{\rho(1-\theta)} \\
& J_{2}=\frac{E_{0}^{1-\nu}}{(1-\nu)[\rho-(1-\nu)(\mu-\eta \varphi)]}-\frac{1}{(1-\nu) \rho}
\end{aligned}
$$

In order to simplify the analysis, in the following we assume $\epsilon=\frac{1}{2}$ : this assumption does not affect the generality of the model but has the advantage to allow for the determination of an explicit expression for $\xi_{2}$. Indeed, $\xi_{2}$ is the implicit solution of the following equation:

$$
g\left(\xi_{2}\right)=\frac{\beta}{\epsilon}+\xi_{2}\left[\mu \epsilon-\frac{1}{2} \sigma^{2} \epsilon(1-\epsilon)-\rho+(1-\epsilon) \eta^{-\frac{\epsilon}{1-\epsilon}} A^{\frac{1}{1-\epsilon}} \xi_{2}^{-\frac{1}{1-\epsilon}}(1-\theta)^{\frac{1}{1-\epsilon}} \xi_{1}^{\frac{1}{1-\epsilon}}\right]=0,
$$

which whenever $\epsilon=\frac{1}{2}$ reduces to a second order equation, with a positive and a negative solution. The positive one is given by:

$$
\xi_{2}=\frac{-4 \beta-\sqrt{4 \beta^{2}-4\left(\mu-\frac{\sigma^{2}}{4}-\rho\right) \mu^{-1} A^{2}(1-\theta)^{2} \xi_{1}^{2}}}{2\left(\mu-\frac{\sigma^{2}}{4}-\rho\right)}
$$

\footnotetext{
${ }^{12}$ From equation $\sqrt{17}$ it also follows that $\mathbb{E}\left[c(t)^{1-\theta}\right] \leq \bar{c}(t)^{1-\theta}$; understanding how $1-u$ is affected by uncertainty is more complex, since from (18), it depends nonlinearly on the random variables $E$ and $K$.

${ }^{13}$ Note that whenever abatement is possible environmental uncertainty and production uncertainty are to some extent similar, since abatement allows somehow to convert production into environmental quality. Despite such a similarity directly comparing the results derived in these two different frameworks is not straightforward.
} 
For the sake of determining a qualitative approximation of the efficient frontier, let us normalize all expressions involving parameters and initial conditions to one and determine how the optimal values of $J_{1}$ and $J_{2}$ depend on $\beta$. It is straightforward to show that the following expressions hold true:

$$
\xi_{2}=\beta+\sqrt{\beta^{2}+1}, \quad \varphi=\frac{1}{\beta+\sqrt{\beta^{2}+1}} .
$$

These expressions allow to explicitly determine how $J_{1}$ and $J_{2}$ behave with respect to $\beta$, which is given by the following:

$$
J_{1}=\frac{\frac{1}{\beta+\sqrt{\beta^{2}+1}}}{1+\left(\frac{1}{\beta+\sqrt{\beta^{2}+1}}\right)^{2}} \quad J_{2}=\frac{1}{1+\left(\frac{1}{\beta+\sqrt{\beta^{2}+1}}\right)^{2}}
$$

These two expressions can be used in order to derive the Pareto frontier looking thus separately at each criterion, or also to derive social welfare looking at the scalarized version of the two criteria. Indeed, by eliminating $\beta$ from the above expressions we can obtain the following analytical expression for the efficient frontier: $J_{1}=J_{2} \sqrt{\frac{1}{J_{2}}-1}$ with $\frac{1}{2} \leq J_{2} \leq 1$. Social welfare instead is the scalarized result of the two criteria weighted by the scalaeization parameter $\beta$ : $W=J_{1}+\beta J_{2}$.
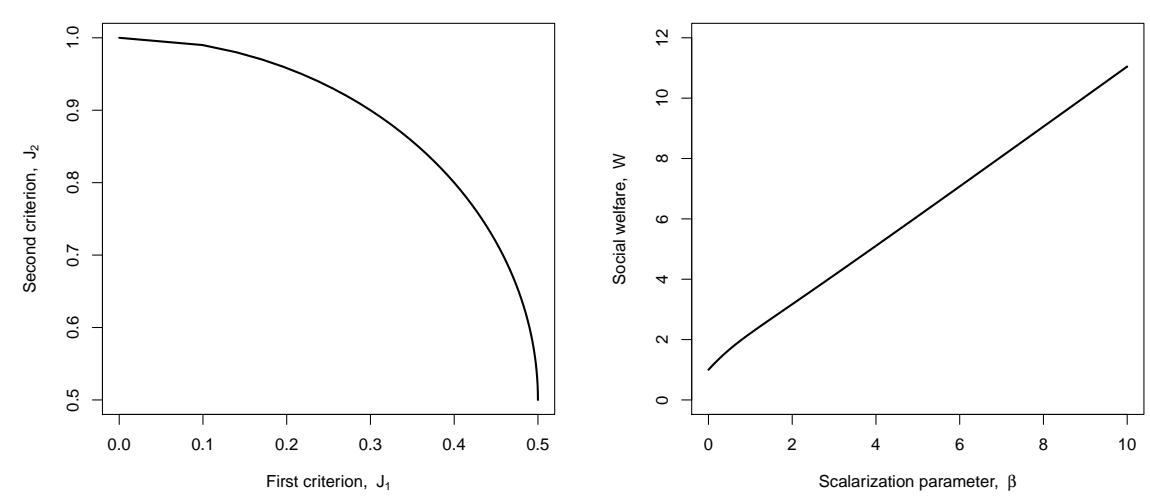

Figure 1: The Pareto frontier (left panel) and social welfare as a function of the scalarization parameter $\beta$ (right panel) in the $\epsilon=\frac{1}{2}$ case.

The left panel in Figure 1 provides a diagrammatic representation of the efficient frontier for our bicriteria problem, which is a traditional growth and environment problem arising in economics, while the right panel shows how the scalarized criterion (representing social welfare in our growth and environment problem) depends on the scalarization parameter. Social welfare is monotoically increasing in $\beta$ which represents the relative weight of the second criterion; this suggests that in order to achieve the highest possible welfare it is desirable to favor the second criterion (i.e., the environmental goal) at the cost of a reduction in the first (i.e., the economic goal), that is moving north-west along the Pareto frontier.

\section{Conclusion}

Apart from trying to bridge the economics and operational research literature by showing how a traditional growth and environment model can be interpreted as a scalarized multicriteria problem, this brief paper tries to shed some light on how the uncertainty about environmental processes affect economic decisions related to abatement activities, and thus economic performance. We show that allocating a certain share 
of output to reduce the costs imposed on the environment by economic growth does not suffice to dampen the negative impacts of uncertainty on both the economy and the environment. Even in the absence of direct productivity effects, once the optimal amount of resources is allocated to environmental preservation activities, net production results enhanced by an indirect productivity effect, which transforms environmental quality into the engine of long run growth. However, even such a substitution of a polluting (capital) with a green (environment) growth source does not prevent uncertainty to restrain economic and environmental performance.

Our results represent only a first attempt to analyze the economic and environmental implications of uncertainty, thus cannot be considered exhaustive; moreover, since they are derived from ad hoc restrictions (like the concavity of the cost function, the low consumption intertemporal elasticity of substitution and the separability of the utility function) our ability to fully describe the implications between growth and environment is only limited. Further investigations are definitely needed in order to improve the explicative power of our model and in order to consider more detailed and complex characterizations of uncertainty and bilateral economy-environment feedbacks. This is left for future research.

\section{A Technical Appendix}

By postulating a value function separable in the state variables of the problem:

$$
J(k, E)=\xi_{1} k^{\lambda_{1}}+\xi_{2} E^{\lambda_{2}}+\xi_{3},
$$

and substituting the relevant expression in the HJB equation, 15 can be rewritten as:

$$
\begin{aligned}
0= & \frac{\theta}{1-\theta}\left[\lambda_{1} \xi_{1}\right]^{\frac{\theta-1}{\theta}} k^{\frac{(\theta-1)\left(\lambda_{1}-1\right)}{\theta}}+\beta \frac{E_{t}^{1-\nu}}{1-\nu}+\xi_{1} k^{\lambda_{1}}[-\delta-\rho]+\xi_{2} E^{\lambda_{2}}\left[\mu \lambda_{2}+\frac{1}{2} \sigma^{2} \lambda_{2}\left(\lambda_{2}-1\right)-\rho\right]+ \\
& -\epsilon^{-\frac{\epsilon}{\epsilon-1}}(\epsilon-1) \eta^{\frac{\epsilon}{\epsilon-1}} A^{-\frac{1}{\epsilon-1}}\left[\lambda_{2} \xi_{2}\right]^{\frac{\epsilon}{\epsilon-1}}\left[\lambda_{1} \xi_{1}\right]^{-\frac{1}{\epsilon-1}} E^{\frac{\epsilon\left(\lambda_{2}-1\right)}{\epsilon-1}} k^{\frac{\phi \epsilon-\lambda_{1}}{\epsilon-1}}-\left[\xi_{3} \rho+\frac{1-\nu+\beta(1-\theta)}{(1-\theta)(1-\nu)}\right]
\end{aligned}
$$

Suppose now $\lambda_{1}=1-\theta, \epsilon \phi=\lambda_{1}=1-\theta$ and $\lambda_{2}=\epsilon=1-\nu$; then we will have:

$$
\begin{aligned}
0= & \xi_{1} k^{1-\theta}\left[\theta(1-\theta)^{-\frac{1}{\theta}} \xi_{1}^{-\frac{1}{\theta}}-\delta-\rho\right]+E^{\epsilon}\left\{\frac{\beta}{\epsilon}+\xi_{2}\left[\mu \epsilon+\frac{1}{2} \sigma^{2} \epsilon(\epsilon-1)-\rho+\right.\right. \\
& \left.\left.-(\epsilon-1) \eta^{\frac{\epsilon}{\epsilon-1}} A^{-\frac{1}{\epsilon-1}} \xi_{2}^{\frac{1}{\epsilon-1}}(1-\theta)^{-\frac{1}{\epsilon-1}} \xi_{1}^{-\frac{1}{\epsilon-1}}\right]\right\}-\left[\xi_{3} \rho+\frac{\epsilon+\beta(1-\theta)}{\epsilon(1-\theta)}\right]
\end{aligned}
$$

Therefore, for this equation to be verified at each time, $t=0, \ldots, \infty$, the following parameter values $\xi_{1}, \xi_{2}, \xi_{3}$ need to be determined as shown in Proposition 1. The optimal rules for consumption and share of emission to abate are obtained by replacing $J_{K}$ and $J_{E}$ into equations (13) and (14) with their expressions as just derived. Then by substituting (17) and (18) into the state equations, we get $(19)$ and (20). Finally, the transversality condition requires that $\lim _{t \rightarrow \infty} E\left[e^{-\rho t} J(k, E)\right]=0$, which because of our guess, means:

$$
\lim _{t \rightarrow \infty} e^{-\rho t} E\left[\xi_{1} k(t)^{1-\theta}+\xi_{2} E(t)^{\epsilon}+\xi_{3}\right]=0 .
$$

The last term in the previous equation converges to zero as long as $\rho$ is positive, while a sufficient condition for the first two terms to converge to zero is $\rho>\epsilon\left[\mu-\eta \varphi-\frac{\sigma^{2}}{2}(1-\epsilon)\right]$. Note that the unique role of this technical condition is ensuring that the transversality condition is verified.

The results of Proposition 2 follows from the following facts: $g(0)=0, \lim _{\xi_{2} \rightarrow+\infty} g\left(\xi_{2}\right)=-\infty$, and $g^{\prime}\left(\xi_{2}\right)<0$. This implies the existence of a unique zero of the function $g$ over $\mathbb{R}_{+}$. Simple calculations show that $\frac{\partial \xi_{2}}{\partial \sigma}<0$.

The results of Proposition 5 are straightforward by computing the expected value of $E(t)^{\epsilon}$ :

$$
\mathbb{E}\left[E(t)^{\epsilon}\right]=E_{0}^{\epsilon} e^{\epsilon(\mu-\eta \varphi) t}
$$


and noting that $\epsilon<1$. This means $\mathbb{E}\left[E(t)^{\epsilon}\right] \leq \bar{E}(t)^{\epsilon}$ and it also implies that $\mathbb{E}\left[k(t)^{1-\theta}\right] \leq \bar{k}(t)^{1-\theta}$. Given the imposed parametric restrictions, the welfare turns out to be:

$$
W=\int_{0}^{\infty}\left[\frac{\left(\frac{\rho+\delta}{\theta}\right)^{1-\theta} k(t)^{1-\theta}-1}{1-\theta}+\beta \frac{E(t)^{\epsilon}-1}{\epsilon}\right] e^{-\rho t} d t
$$

since it is a linear combination of $k(t)^{1-\theta}$ and $E(t)^{\epsilon}$, it then follows that $\mathbb{E}[W] \leq \bar{W}$. Note that in order to determine the optimal level of welfare $W$ we need the optimal values of both criteria $J_{1}$ and $J_{2}$; these values can be obtained by plugging the optimal trajectories of the state and control variables into the relevant criterion, as follows:

$$
\begin{aligned}
& J_{1}:=\mathbb{E}\left[\int_{0}^{\infty}\left(\frac{\left(\frac{\rho+\delta}{\theta}\right)^{1-\theta} k(t)^{1-\theta}-1}{1-\theta}\right) e^{-\rho t} d t\right] \\
& J_{2}:=\mathbb{E}\left[\int_{0}^{\infty}\left(\frac{E(t)^{1-\nu}-1}{1-\nu}\right) e^{-\rho t} d t\right]
\end{aligned}
$$

Tedious algebra allows to calculate the following expressions for the optimal value of the two criteria:

$$
\begin{aligned}
J_{1} & =\frac{\left(\frac{\rho+\delta}{\theta}\right)^{1-\theta} E_{0}^{\epsilon} A \varphi^{\epsilon}}{[\rho-\epsilon(\mu-\eta \varphi)][(1-\theta) \Psi+\rho]}+\frac{\left(\frac{\rho+\delta}{\theta}\right)^{1-\theta} E_{0}^{\epsilon} k_{0}^{1-\theta}}{(1-\theta)[(1-\theta) \Psi+\rho]}-\frac{1}{\rho(1-\theta)} \\
J_{2} & =\frac{E_{0}^{1-\nu}}{(1-\nu)[\rho-(1-\nu)(\mu-\eta \varphi)]}-\frac{1}{(1-\nu) \rho}
\end{aligned}
$$

which imply that the the optimal value of welfare $W$ is given by:

$$
\begin{aligned}
W= & \frac{\left(\frac{\rho+\delta}{\theta}\right)^{1-\theta} E_{0}^{\epsilon} A \varphi^{\epsilon}}{[\rho-\epsilon(\mu-\eta \varphi)][(1-\theta) \Psi+\rho]}+\frac{\left(\frac{\rho+\delta}{\theta}\right)^{1-\theta} E_{0}^{\epsilon} k_{0}^{1-\theta}}{(1-\theta)[(1-\theta) \Psi+\rho]}+\frac{\beta E_{0}^{1-\nu}}{(1-\nu)[\rho-(1-\nu)(\mu-\eta \varphi)]}+ \\
& -\frac{1}{\rho(1-\theta)}-\frac{\beta}{(1-\nu) \rho}
\end{aligned}
$$

\section{References}

1. Aghion, P., Angeletos, G.M., Banerjee, A., Manova, K. (2010). Volatility and growth: credit constraints and the composition of investment. Journal of Monetary Economics 57, 246-265

2. Andreoni, J., Levinson, A. (2001). The simple analytics of the environmental Kuznets curve, Journal of Public Economics 80, 269-286

3. Aouni, B., Colapinto, C., La Torre, D., Liuzzi, D., Marsiglio, S. (2015). On dynamic multiple criteria decision making models: a goal programming approach, in (Al-Shammari, M., Masri, H., Eds)) "Multiple Criteria Decision Making in Finance, Insurance and Investment", 31-48 (Springer International Publishing: Switzerland)

4. Athanassoglou, S., Xepapadeas, A. (2012). Pollution control with uncertain stock dynamics: when, and how, to be precautious, Journal of Environmental Economics and Management 63, 304-320

5. Bartz, S., Kelly, D.L. (2008). Economic growth and the environment: theory and facts, Resource and Energy Economics 30, 115-149

6. Brock, W.A., Taylor, M.S. (2005). Economic growth and the environment: a Review of theory and empirics, in (Aghion P., Durlauf, S., Eds.) "Handbook of Economic Growth", vol.1 (Elsevier:Amsterdam, Netherlands)

7. Bucci, A., Colapinto, C., Forster, M., La Torre, D. (2011). Stochastic technology shocks in an extended Uzawa-Lucas model: closed-form solution and long-run dynamics, Journal of Economics 103, 83-99 
8. Chevé, M. (2000). Irreversibility of pollution accumulation, Environmental and Resource Economics $16,93-104$

9. Colapinto, C., Liuzzi, D., Marsiglio, S. (2015). Sustainability and intertemporal equity: a multicriteria approach, Annals of Operations Research, forthcoming, DOI: 10.1007/s10479-015-1837-1

10. Dasgupta, P., Heal, G.M. (1978). Economic theory and exhaustible resources (Cambridge University Press: Cambridge)

11. De Hek, P.A. (1999). On endogenous growth under uncertainty, International Economic Review 40, $727-744$

12. Kijima, M., Nishide, K., Ohyama, A. (2011). EKC-type transitions and environmental policy under pollutant uncertainty and cost irreversibility, Journal of Economic Dynamics \& Control 35, 746-763

13. La Torre, D., Liuzzi, D., Marsiglio, S. (2016). Pollution control under uncertainty and sustainability concern, Environmental and Resource Economics, forthcoming, DOI: 10.1007/s10640-016-0010-x

14. Marsiglio, S., La Torre, D. (2012). A note on demographic shocks in a multi-sector growth model, Economics Bulletin 32, 2293-2299

15. Marsiglio, S. (2014). Reassessing Edgeworth's conjecture when population dynamics is stochastic, Journal of Macroeconomics 42, 130-140

16. Mohtadi, H. (1996). Environment, growth, and optimal policy design, Journal of Public Economics 63, 119-140

17. Privileggi, F., Marsiglio, S. (2013). Environmental shocks and sustainability in a basic economyenvironment model, International Journal of Applied Nonlinear Science 1, 67-75

18. Ramsey, F. (1928). A mathematical theory of saving, Economic Journal 38, 543-559

19. Ricci, F. (2007). Channels of transmission of environmental policy to economic growth: a survey of the theory, Ecological Economics 60, 688-699

20. Sawaragi, Y., Nakayama, H., Tanino, T. (1985). Theory of multiobjective optimization (Academic Press, Inc.)

21. Shieh, J.Y., Lai, C.C., Chen, J.H. (2001). A comment on Huang and Cai's constant-returns endogenous growth with pollution control, Environmental and Resource Economics 20, 165-172

22. Smith, W.T. (2007). Inspecting the mechanism exactly: closed-form solution to a stochastic growth model, The B.E. Journal of Macroeconomics 7 (Contributions), Article 30

23. Smulders, S. (1999). Endogenous growth theory and the environment. In: van den Bergh, J. (Ed.), The Handbook of Environmental and Resource Economics (Edward Elgar: Cheltenham)

24. Soretz, S. (2003). Stochastic pollution and environmental care in an endogenous growth model, Manchester School 71, 448-469

25. Soretz, S. (2004). Stochastic environmental policy, risk-taking, and growth: discretion versus commitment, International Journal of Global Environmental Issues 4, 53-71

26. Soretz, S. (2007). Efficient dynamic pollution taxation in an uncertain environment, Environmental and Resource Economics 36, 57-84

27. Steger, T.M. (2005). Stochastic growth under Wiener and Poisson uncertainty, Economics Letters 86, 311-316

28. Stokey, N.L. (1996). Are there limits to growth?, International Economic Review 39, 1-31

29. United Nations Environment Programme (2012). The future we want - Rio+20 outcome document, available on-line at: http://www.uncsd2012.org/thefuturewewant.html

30. Walde, K. (2005). Endogenous growth cycles, International Economic Review 46, 867 -894

31. Xepapadeas, A. (2005). Economic growth and the environment. In: M ler, K.-G., Vincent, J. (Eds.), Handbook of Environmental Economics, vol. 3., 1219-1271 (Elsevier: Amsterdam, Netherlands) 